\title{
Narcisismo manifiesto, narcisismo encubierto y trastornos de personalidad en una Unidad de Conductas Adictivas: validez predictiva de respuesta a tratamiento \\ Open Narcissism, covered narcissism and personality disorders as predictive factors of treatment response in an out-patient Drug Addiction Unit
}

\author{
José Salazar-Fraile*; Carmen Ripoll-Alandes**; \\ JULIO BOBES ${ }^{\star \star \star}$
}

\author{
* Centro de Salud Mental de Paterna, Unidad de \\ Psicopatología Neurofisiológica. Departamento de Salud 6. Conselleria \\ de Sanitat. Generalitat Valenciana. Centro de Investigación Biomédica en \\ Red de Salud Mental (CIBERSAM). \\ ** Unidad de Conductas Adictivas de Campanar. Departamento de \\ Salud 7. Conselleria de Sanitat. Generalitat Valenciana. \\ *** Area de Psiquiatría. Universidad de Oviedo. Centro de Investigación \\ Biomédica en Red de Salud Mental (CIBERSAM).
}

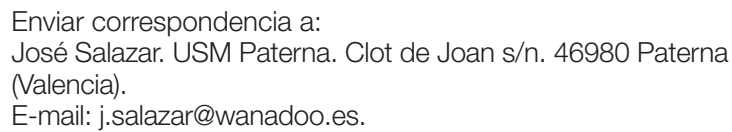

\section{RESUMEN}

Introducción. Aunque se ha estudiado ampliamente la prevalencia de los trastornos de personalidad en la población de usuarios de drogas, la literatura sobre su valor predictivo para el tratamiento es controvertida. Por otro lado, respecto a los rasgos de personalidad, aunque también se ha estudiado su valor predictivo para el tratamiento, son escasos los estudios sobre la validez de los rasgos de personalidad narcisista. Objetivos. Estudiar el valor predictivo de los trastornos de personalidad y de rasgos de personalidad relacionados con el narcisismo y la autoestima en la respuesta a tratamiento de abuso de drogas. Método. Se entrevistaron 78 pacientes atendidos en una Unidad de Conductas Adictivas evaluando la existencia de trastorno de personalidad asi como medidas de autoestima, narcisismo y narcisismo encubierto (hipersensible). Estas variables se emplearon como predictoras de recaída en el consumo de la droga principal en un modelo de supervivencia de regresión de Cox. Resultados. Los diagnósticos de trastorno Limite y Pasivo-agresivo de la personalidad así como el narcisismo hipersensible (encubierto) presentaron mayor riesgo de recaidas mientras que el narcisismo manifiesto presentó un carácter protector. La autoestima no mostró valor predictivo. Conclusiones. Respecto a los trastornos de personalidad, los pacientes caracterizados por la impulsividadinestabilidad y la pasividad-resentimiento presentan mayor riesgo de recaida durante el tratamiento. Respecto a los rasgos de personalidad, la sensibilidad excesiva a la humillación supone un factor de riesgo de recaida mientras que el orgullo y la confianza en sí mismo supone un factor de protección.

Palabras clave: narcisismo, autoestima, respuesta a tratamiento, uso de sustancias, trastorno de personalidad.

\section{ABSTRACT}

Introduction. Although a high prevalence of personality disorders has been reported in substance users, the literature on their value for predicting treatment response is controversial. On the other hand, while the predictive validity of personality traits as predictors of response to drug abuse or dependence has been studied, research on the validity of narcissistic personality traits is scarce. Objectives. To study the predictive value of personality disorders, narcissistic personality traits and selfesteem for predicting treatment response. Method. We assessed 78 patients attended at an addiction treatment unit using personality disorder diagnoses and measures of self-esteem, narcissism and covert (hypersensitive) narcissism. These variables were used in a Cox survival model as predictive variables of time to relapse into drug use. Results. Hypersensitive (covert) narcissism and borderline and passive-aggressive personality disorders were risk factors for relapse into drug use, while open narcissism was a protective factor. Self-esteem did not show predictive validity. Conclusions. Personality disorders characterized by impulsivity-instability and passivity-resentfulness show higher risk of relapse into drug abuse. Personality traits characterized by high sensitivity to humiliation increase the risk of relapse, whereas pride and self-confidence are protective factors.

Key words: narcissism, self-esteem, treatment response, substance use, personality disorders. 


\section{INTRODUCCIÓN}

os estudios de pronóstico de respuesta a tratamiento de abuso o dependencia de drogas se caracterizan -por la obtención de resultados muy diversos y con frecuencia contradictorios (Poling, Kosten, y Sofuoglu, 2007; Stotts, Mooney, Sayre, Novy, Schmitz, y Grabowski, 2007). Las variables predictivas estudiadas se caracterizan por una diversidad elevada comprendiendo las sociodemográficas, familiares, legales, comorbilidad con otras patologías asi como caracterización del consumo, de la adicción o de la abstinencia. Entre las variables clínicas destacan las referidas a rasgos de personalidad y los trastornos de personalidad, especialmente el grupo B del Eje II de la DSM-IV (American Psychiatric Association [APA], 1994; Poling et al., 2007; Stotts et al., 2007).

Respecto a los trastornos de personalidad, numerosos estudios clínicos y epidemiológicos han mostrado una prevalencia elevada de trastornos de personalidad en poblaciones con trastorno por abuso o dependencia de drogas. Aunque también se citan otros trastornos de personalidad, los más prevalentes son los integrantes del grupo B (límite, antisocial, narcisista e histriónico), el pasivo-agresivo y el paranoide (para una revisión véase López-Durán y BecoñaIglesias, 2006). Sin embargo, son escasos y con frecuencia con resultados controvertidos, los estudios sobre el valor predictivo de los trastornos de personalidad como variables pronósticas de adherencia y respuesta a tratamiento (Pettinati, Pierce, Belden, y Meyers, 1999; Stotts et al., 2007). Respecto a los rasgos de personalidad se ha descrito su valor predictivo de respuesta al tratamiento, especialmente en los rasgos de impulsividad y de búsqueda de sensaciones (Lemus, Downey, Arfken, Henderson, y Schuster, 2001; Moeller, Dougherty, Barrat, Schmitz, Swann, y Grabowski, 2001; Roll, Saules, Chudzynski, y Sodano, 2004). Si bien algunos de estos rasgos como la impulsividad forman un componente importante de algunos trastornos de personalidad como el trastorno límite, existen otros rasgos de personalidad como el narcisismo o la autoestima, también importantes en trastornos de personalidad del grupo $B$, de los que se carece de estudios sobre su valor predictivo de respuesta a tratamiento. Los rasgos de narcisismo encubierto o hipersensible se presentan con mayor intensidad en los trastornos de personalidad límite, narcisista y pasivo-agresivo (Ronningstam, 2005; Ripoll, Salazar, y Bobes, en prensa) mientras que, por otro lado, los rasgos de narcisismo manifiesto se presentan con mayor intensidad en los trastornos narcisista y antisocial de la personalidad (Horowitz, 2004; Ronningstam, 2005).

Los trastornos de personalidad se caracterizan por una percepción distorsionada de sí mismo así como un patrón no adaptativo de relaciones interpersonales y de control de impulsos. Entre los trastornos de personalidad, los trastornos del grupo B comparten la caracteristica de un patrón disfuncional de relaciones interpersonales y una tendencia a la percepción sobredimensionada o inestable de sí mismo (Millon y Davis 1996). Sin embargo, cuando se estudian los distintos trastornos de personalidad como factores predictivos de la respuesta al tratamiento de las conductas adictivas no se suele distinguir entre la influencia de los rasgos de personalidad y los trastornos de personalidad. Resulta para ello importante la discriminación de la contribución relativa de estas dos dimensiones (trastorno de personalidad y personalidad), relacionadas pero independientes, pues la respuesta a tratamiento de los trastornos adictivos podría ser explicada por cualquiera de los dos factores. En el presente trabajo analizamos el valor predictivo de la autoestima y el narcisismo junto con los trastornos de personalidad como factores de riesgo en la recaída del tratamiento ambulatorio para desintoxicación de drogas.

\section{MÉTODO}

\section{Muestra}

Se solicitó la participación en el estudio a 79 pacientes drogodependientes atendidos consecutivamente en la Unidad de Conductas Adictivas Valencia 07 de la Conselleria de Sanitat de Valencia que cumplian los criterios de inclusión de edad comprendida entre 18-65 años y diagnosticados de abuso o dependencia de drogas según criterios DSMIV. Del total de los sujetos, 26 fueron mujeres (33\%) y 53 hombres (67\%). La edad media fue de 32,5 años para las mujeres y 36,6 años para los hombres (amplitud $=23-52$ ), con más años de escolarización en las mujeres $(11,5)$ que en los hombres $(10,5)$. El $81 \%$ de la muestra se encontró en situación laboral de activo. Algo más de la mitad de los pacientes estaban solteros, mientras que una cuarta parte se encontraban separados o divorciados. En cuanto al diagnóstico de trastorno por consumo de sustancias, algo más de la mitad de la muestra tenía una dependencia de cocaína $(51,9 \%)$ y un tercio $(29,1 \%)$ dependencia de opiáceos. Se consiguió el seguimiento de 78 pacientes con un porcentaje de seguimiento del $98 \%$.

\section{Procedimiento}

Se excluyeron los pacientes con sintomatología orgánica, psicótica o afectiva que pudiera condicionar la información proporcionada. Los pacientes proporcionaron el consentimiento informado para la participación en el estudio y el diseño fue aprobado por el Comité Científico y Ético de la Fundación para la Investigación del Hospital La Fe de Valencia. Se definió como respuesta a tratamiento la ausencia de consumo de la droga principal medida mediante urinoanálisis semanal durante el intervalo de seis meses desde el momento de su inclusión en el estudio. Junto con las variables sociodemográficas los participantes en el estudio cumplimentaron los siguientes instrumentos en la primera entrevista:

Escala de Narcisimo Hipersensible. Se empleó la versión española de la Hypersensitive Narcissism Scale (HSNS, Hending y Cheek 1997). Esta escala valora el narcisismo hipersensible o encubierto y su versión española ha mostrado unas propiedades psicométricas satisfactorias en su consistencia interna (alfa de Cronbach $=0,73$ ), fiabilidad 
test-retest (Coeficiente de Correlación Intraclase $=0,67$ ) así como en su validez factorial y discriminante, mostrando independencia del nivel de instrucción y la edad (Ripoll et al., en prensa).

Escala de Personalidad Narcisista (EPN). Para evaluar el narcisismo manifiesto se empleó la versión española de la Narcissistic Personality Inventory (NPI, Raskin y Terry 1988; García y Cortés, 1998).

Escala de Autoestima Global de Rosenberg (EAR). Se empleó la versión española de la Global Self-Esteem Scale (Rosenberg, 1973; Vázquez Morejón, Jiménez Garcia-Bóveda, y Vázquez-Morejón Jiménez, 2004).

Entrevista Clínica Estructurada para los Trastornos de la Personalidad del EJE II del DSM-IV. Para el diagnóstico de los trastornos de personalidad se empleó la versión española de la SCID-II (Gómez-Beneyto, Villar, Renovell, Pérez, Hernández, Cuquerella, et al., 1994). Se cumplimentaron los trastornos de personalidad en los que el rasgo de narcisismo manifiesto o el de narcisismo hipersensible presentan mayor intensidad contribuyendo significativamente a su constructo (Horowitz, 2004; Ronningstam, 2005): histriónico, narcisista, límite, antisocial y trastorno pasivo-agresivo de la personalidad. Se consideró el trastorno antisocial de la personalidad en aquellos sujetos que cumplieron los criterios de trastorno antisocial de la personalidad en la infancia y edad adulta. Los sujetos que solo cumplieron los criterios diagnósticos en la edad adulta fueron considerados como comportamiento antisocial.

Análisis. Tras el estudio descriptivo, se llevó a cabo un análisis de supervivencia mediante el método de KaplanMeier para estimar si el tiempo de recaída en el consumo era significativamente distinto en los sujetos con trastorno de personalidad respecto a los que no tenían trastorno. Para el análisis multivariante de los trastornos y rasgos de personalidad estudiados se empleó posteriormente un modelo de análisis de supervivencia mediante Regresión de Cox. En este modelo, como variables independientes se emplearon las variables categóricas de trastorno de personalidad pasivo agresivo, antisocial, histriónico, límite, narcisista y comportamiento antisocial junto con las variables cuantitativas obtenidas con la puntuación total de las escalas EPN, HSNS y EAR. Para la variable dependiente, el acontecimiento se definió como el consumo de la droga principal de modo que la ecuación expresara el riesgo de no responder al tratamiento. Se utilizó un procedimiento de pasos sucesivos hacia delante.

Las curvas de supervivencia se obtuvieron mediante el método de Kaplan-Meier. Se comprobó que se cumplian los requisitos del modelo de Cox de riesgos proporcionales verificando que las curvas generadas por el método log minus log de las funciones de supervivencia eran paralelas para las variables predictivas dicotómicas que resultaron significativas.

\section{RESULTADOS}

Respecto a la sustancia principal estudiada, 41 (52\%) sujetos habían experimentado un episodio de dependencia a lo largo de su vida; 21 (27\%) presentaron dos episodios y
$14(18 \%)$ más de dos episodios. El resto de los participantes (3\%) fue diagnosticado de abuso de substancias. Los participantes presentaron una duración media total 71 meses de dependencia.

Al final del seguimiento 40 sujetos (51\%) permanecieron abstinentes mientras que 38 (48\%) habian recaído. Se experimentó la pérdida de un (1\%) paciente en el seguimiento.

Los pacientes con trastorno de personalidad no se diferenciaron en cuanto al número de días en seguimiento de los pacientes sin trastorno de personalidad (media para los pacientes $\sin$ trastorno $=128$ días, media para los pacientes con trastorno $=127$ días, $t=0,05, p=n s$ ). Tampoco se apreciaron diferencias entre hombres y mujeres en el número de dias de seguimiento. El tiempo de seguimiento tampoco se correlacionó con el número de episodios de abuso o dependencia o con el tiempo de dependencia o de abuso. Finalmente, los pacientes abstinentes no se diferenciaron de los no abstinentes en cuanto al número de episodios y la duración de la dependencia o del abuso.

Aunque los pacientes con trastorno de personalidad presentaron un tiempo medio de recaída en el consumo (158 dias) menor que los pacientes sin trastorno de personalidad (182) días, la diferencia entre ambos tiempos no fue significativa (log rank, Mantel-Cox $\chi^{2}=1,2, p=n s$ ).

El modelo de supervivencia de Cox mostró que los pacientes con trastorno pasivo-agresivo de la personalidad presentaron un riesgo de recaída en el consumo tres veces y medio superior a quienes no lo presentaron y los pacientes con trastorno límite de la personalidad presentaron un riesgo casi cuatro veces mayor. El narcisismo encubierto o hipersensible resultó un factor de riesgo de recaída en el consumo mientras que el narcisismo manifiesto supuso un factor de protección $\left(\chi^{2}\right.$ del modelo $\left.=22,408, p<0,01\right)$. El modelo no consideró significativos para el riesgo de recaídas en el consumo la presencia de trastorno de personalidad histriónico, antisocial o narcisista, ni tampoco el comportamiento antisocial adulto (Tabla 1). Las figuras 1 y 2 muestran las curvas de supervivencia en función de la presencia o ausencia del diagnóstico de trastorno de personalidad pasivo-agresivo y límite.

Tabla 1. Modelo de regresión de Cox. Razones de riesgo de consumo para los trastornos de personalidad indicados, comportamiento antisocial adulto, autoestima, narcisismo manifiesto y narcisismo encubierto.

\begin{tabular}{lcccc}
\hline & B & $p$ & $\operatorname{Exp}(\beta)$ & IC del 95\% para $\operatorname{Exp}(\beta)$ \\
\hline Trastorno Pasivo-agresivo & 1,273 & 0,045 & 3,571 & $1,031-12,372$ \\
Trastorno Histriónico & 0,408 & 0,538 & 1,504 & $0,411-5,512$ \\
Trastorno Narcisista & $-0,846$ & 0,316 & 0,429 & $0,082-2,245$ \\
Trastorno Limite & 1,372 & 0,011 & 3,942 & $1,362-11,407$ \\
Trastorno Antisocial & 0,143 & 0,759 & 1,154 & $0,464-2,871$ \\
Comportamiento Antisocial Adulto & 0,375 & 0,522 & 1,456 & $0,461-4,596$ \\
Narcisismo Encubierto & 0,145 & 0,003 & 1,155 & $1,051-1,270$ \\
Narcisismo Manifiesto & $-0,090$ & 0,023 & 0,914 & $0,846-0,988$ \\
Autoestima & 0,131 & 0,083 & 1,140 & $0,983-1,321$ \\
\hline
\end{tabular}

$\chi^{2}$ del modelo $=22,408, p<0,01$

$\mathrm{IC}=$ intervalo de confianza. 


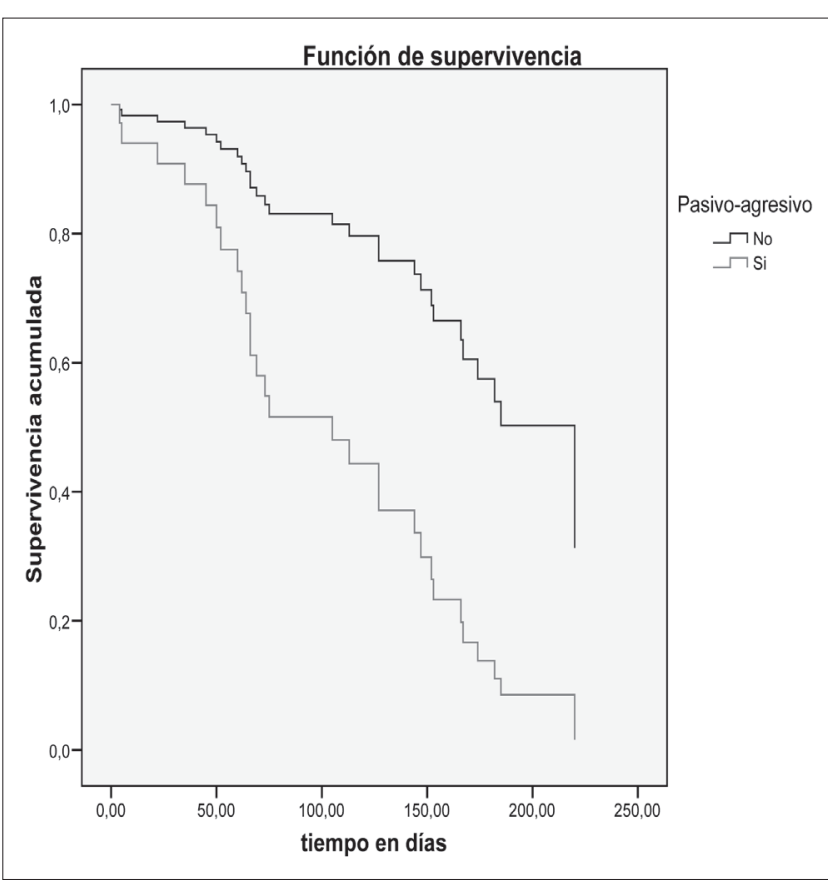

Supervivencia acumulada = incidencia acumulada

Figura 1. Riesgo de consumo para el trastorno de personalidad pasivo-agresivo.

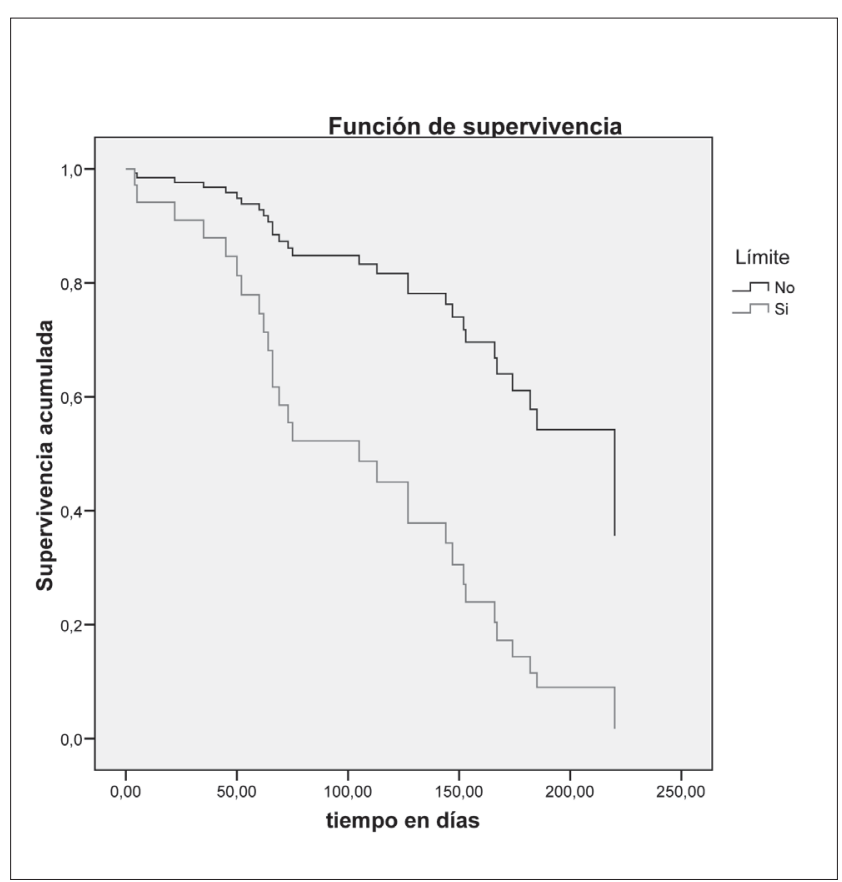

Supervivencia acumulada = incidencia acumulada

Figura 2. Riesgo de consumo para el trastorno límite de personalidad.

\section{DISCUSIÓN}

El presente trabajo ha estudiado el valor predictivo de los trastornos de personalidad del Cluster B y el Trastorno Pasivo Agresivo junto con la dimensión narcisista de la personalidad como factores pronósticos de adherencia y respuesta a tratamiento en una muestra de 78 pacientes atendidos en una consulta ambulatoria para el tratamiento por abuso o dependencia de drogas. El factor general Trastorno de Personalidad no ha resultado predictivo de la adherencia a tratamiento, habiéndolo sido en cambio la presencia de los trastornos específicos de Trastorno de Personalidad Límite y Trastorno de Personalidad Pasivo-Agresivo.

El riesgo de consumo de sustancias debido al Trastorno Límite ha sido ampliamente descrito, siendo uno de los criterios diagnósticos de este trastorno la presencia de comportamientos impulsivos, entre ellos el consumo de drogas (APA, 1994). Resulta perfectamente coherente que los pacientes con este tipo de personalidad asociada a la impulsividad presenten una peor respuesta a tratamiento, con un riesgo de recaída cuatro veces superior a quienes no lo padecen. En el caso del Trastorno Pasivo-Agresivo, no suele ser un trastorno estudiado con frecuencia en la literatura. El rencor y la pasividad definitorios de este tipo de trastorno (APA, 1994) explicarian el riesgo de recaída en el consumo. Por un lado la susceptibilidad a experimentar estados de ánimo intensos y encubiertos como el rencor (Millon y Davis 1996) llevaría a la necesidad de moderarlos mediante el consumo de drogas, y por otro lado la pasividad (Millon y Davis 1996) determi- naría la ausencia de una actitud o compromiso activo en el tratamiento, condicionando con ello una alta probabilidad de recaida en el consumo.

Respecto a las distintas dimensiones del narcisismo, apreciamos que la autoestima no representa un factor ni de protección ni de riesgo para las recaídas en el consumo. Sin embargo el narcisismo presenta un factor de protección reduciendo el riesgo de recaída. En consonancia con nuestro estudio, se ha descrito ampliamente el narcisismo manifiesto como factor motivador en la persistencia y en la consecución de metas (Ronningstam, 2005), especialmente cuando suponen posibilidad de auto-enaltecimiento e incremento de reconocimiento social (Wallace y Baumeister 2002). Además, otros autores han descrito el valor motivacional del sentimiento de orgullo, característica básica del narcisismo, en la perseverancia para el logro de objetivos (Williams y DeSteno 2008). En este sentido, el orgullo y el reconocimiento resultarian factores motivadores para la persistencia en una meta, en este caso la de la abstinencia.

En nuestro estudio, el narcisismo encubierto o hipersensible ha mostrado ser un factor de riesgo para la recaída en el consumo de sustancias. En contraste con el narcisismo extrovertido, el narcisismo hipersensible (encubierto) reduce las posibilidades de conseguir metas difíciles pues la auto-regulación emocional se encuentra condicionada por la vergüenza (Ronningstam 2005). La vergüenza implica una evaluación negativa de la percepción de sí mismo (Tangney y Salovey 1999), un aumento de la sensibilidad a las evaluaciones negativas (Tangney y Salovey 1999), una distorsión en la 
autoevaluación de los procesos de autorregulación intrapersonal (Rodewalt y Sorrow 2003) y mecanismos de defensa para manejar la emoción desagradable generalmente caracterizados por la rabia y la evitación de la situación causante de dicha emoción (Tangney y Salovey 1999). La combinación de estos factores dificulta el mantenimiento de la abstinencia al interferir en procesos implicados en la autorregulación para el control del consumo de substancias tales como el establecimiento de metas adecuadas y la auto-observación (Sayete 2004). Además, el manejo de emociones intensas requiere un esfuerzo, muchas veces sostenido, que finalmente puede implicar un agotamiento de recursos personales llevando consigo el abandono en la persistencia (Baumeister, Bratslavsky, Muraven y Tice 1998; Dale y Baumeister 1999).

Diferencias en las características de las poblaciones de referencia de las muestras estudiadas, así como en las metodologías empleadas en el diagnóstico y análisis (generalmente a posteriori) de los factores predictivos, explican la gran discrepancia de resultados en el estudio de los trastornos de personalidad como predictores de respuesta a tratamiento (Poling et al., 2007; Stotts et al., 2007). En nuestro caso, al igual que en otros estudios (McKay, Alterman, Cacciola, Mulvaney, y O'Brien, 2000; Pettinati et al., 1999), resalta la ausencia de riesgo asociada con el trastorno antisocial de personalidad. En este sentido, la carencia de empatía en combinación con el egoismo, características fundamentales del trastorno antisocial, no representarian un factor de riesgo en la población atendida en nuestro ámbito.

Nuestros resultados pueden encontrarse limitados a los pacientes atendidos en un centro de salud mental público y en el contexto del área de cobertura de la población de referencia del centro donde se ha llevado a cabo el estudio. Sería arriesgado extrapolar estos hallazgos a todo tipo de población atendida por consumo de drogas. Por ello resultan imprescindibles estudios posteriores para dilucidar el papel del narcisismo y el narcisismo hipersensible en otros ámbitos que supongan características sociodemográficas, clínicas y legales diferentes a nuestra muestra.

En conclusión, el presente estudio ha mostrado que en el tratamiento de la adicción o abuso de drogas es necesario considerar no solo la presencia de trastorno límite de la personalidad sino también la existencia del trastorno pasivo agresivo. De este modo, comportamientos de resistencia a satisfacer las expectativas de tratamiento tales como obstinación o postergación son más fácilmente interpretables como resultado de sentimientos de resentimiento o de búsqueda de gratificación en el contexto de un trastorno de personalidad. Además, también deberían tenerse en cuenta en la planificación y en el análisis de la interacción del paciente con el profesional rasgos de personalidad asociados a estos trastornos, pero no equivalentes con ellos. La utilización de metas marcadas por retos en los que se implique el sentimiento de orgullo puede ejercer un factor motivador potente en los sujetos con un narcisismo manifiesto. Sin embargo, en sujetos con rasgos de narcisismo hipersensible, la elevada sensibilidad a la humillación y el fracaso serán factores a ser tenidos en cuenta a la hora de establecer metas o formas de control externo o interno.

\section{REFERENCIAS}

American Psychiatric Association. (1994). Diagnostic Criteria from DSM-IV. Washington, DC: Autor.

Baumeister, R., Bratslavsky, E., Muraven, M., y Tice, D. (1998). Ego depletion: is the active self a limited resource? Journal of Personality and Social Psychology, 74, 1252-1265.

Dale, K., y Baumeister, R. (1999). Self-Regulation and Psychopathology. En: R. Kowalski y M. Leary (Eds). The Social Psychology of Emotional and Behavioral Problems. Washington, DC. American Psychological Association.

García, J. M., y Cortés, J. F. (1998). La medición empírica del narcisismo Psicothema, 10, 725-735.

Gómez-Beneyto, M., Villar, M., Renovell, M., Pérez, F., Hernández, M., Cuquerella, M., et al. (1994). The diagnosis of personality disorders with a modified version of the SCID-II in a Spanish clinical sample. Journal of Personality Disorders, 8, 104-110.

Hendin, H. M., y Cheek, J. M. (1997). Assessing Hypersensitive Narcissism: A Re-examination of Murray's Narcissism Scale. Journal of Research in Personality, 31, 588-599.

Horowitz L. (2004). Interpersonal foundations of psychopathology. Washington, DC. American Psychological Association.

Lemus, T. C., Downey, K. K., Arfken, C. L., Henderson, M. J., y Schuster, C. R. (2001). Novelty seeking as a predictor of treatment retention for heroin dependent cocaine users. Drug and Alcohol Dependence, 61, 287-295.

López Durán, A., y Becoña Iglesias, E. (2006). Patrones y trastornos de personalidad en personas con dependencia de la cocaína en tratamiento. Psicothema, 18, 578-583.

McKay, J. R., Alterman, A. I., Cacciola, J. S., Mulvaney, F. D., y O'Brien, Ch. P. (2000). Prognostic significance of antisocial personality disorder in cocaine-dependent patients entering continuing care. Journal of Nervous and Mental Disease 188, 287-296.

Millon, T., y Davis, R. (1996). Disorders of personality DSM-IV and beyond. New York: Willey \&t Sons.

Moeller, F. G., Dougherty, D. M., Barrat, E. S., Schmitz, J. M., Swann, A.C., y Grabowski, J. (2001). The impact of impulsivity on cocaine use and retention in treatment. Journal of Substance Abuse Treatment, 21, 193-198.

Pettinati, H. M., Pierce, J. D. Jr., Belden, P. P., y Meyers, K. (1999). The relationship of Axis II personality disorders to other known predictors of addiction treatment outcome. American Journal on Addictions 8, 136-147.

Poling, J., Kosten, T., y Sofuoglu, M. (2007). Treatment outcome predictors for cocaine dependence. Amercan Journal of Drug and Alcohol Abuse, 33, 91-206.

Ripoll, C., Salazar, J., y Bobes, J. (en prensa). Validez de la versión española de la Hypersensitive Narcissism Scale (HSNS) en una Unidad de Conductas Adictivas. Adicciones.

Raskin, R., y Terry, H. (1988). A principal-components analysis of the narcissistic personality inventory and further evidence of its construct validity. Journal of Personality and Social Psychology, 54, 890-902. 
Rosenberg, M. (1973). La autoimagen del adolescente en la sociedad. Buenos Aires: Paidós.

Rol, J. M., Saules, K. K., Chudzynski, J. E., y Sodano, R. (2004). Relationship between Tridimensional Personality Questionnaire scores and clinic attendance among cocaine abusing, buprenorphine maintained outpatients. Substance Abuse and Misuse, 39, 1025-1040.

Ronningstam, E. (2005). Identifying and understanding the narcissistic personality. Oxford: Oxford University Press.

Sayette, M. A. (2004). Self-regulatory failure and addiction. En: R. Baumeister y K. Vohs (Eds). Handbook of self-regulation. Research, theory and applications. New York. The Guilford Press.

Stotts, A. L., Mooney, M.E., Sayre, S.L., Novy, M., Schmitz, J. M., y Grabowski, J. (2007). Illusory predictors: Generalizability of findings in cocaine treatment retention research. Addictive Behaviors 32, 2819-2836.
Tangney, J., y Salovey, P. (1999). Problematic social emotions: shame, guilt, jealousy, and envy. En: R. Kowalski y M. Leary (Eds). The Social Psychology of Emotional and Behavioral Problems. Washington, DC. American Psychological Association.

Vazquez Morejón, J., Jiménez Garcia-Bóveda, R. y Vázquez-Morejón Jiménez, R. (2004). Escala de Autoestima de Rosenberg: fiabilidad y validez en población clínica española. Apuntes de Psicología, 22, 247-255.

Wallace, H. M., y Baumeister, R. F. (2002). The performance of narcissists rises and falls with perceived opportunity for glory. Journal of Personality and Social Psychology, 82, 819-834.

Williams, L. A., y DeSteno, D. (2008). Pride and perseverance: the motivational role of pride. Journal of Personality and Social Psychology, 94, 1007-1017. 